\title{
A CMOS TRIODE TRANSCONDUCTOR
}

\author{
Bram Nauta, Eric Klumperink, Wim Kruiskamp \\ MESA Research Institute \\ University of Twente
}

PO. Box 217,7500 AE, Enschede, The Netherlands.

phone: $x-3153892643$

fax: $x-3153354003$

\begin{abstract}
A novel versatile CMOS voltage to current converter is presented. The conversion transistors operate in the triode region. Key points are: high linearity ( $T H D<0.4 \%$ for $6 \mathrm{~V}_{\mathrm{pp}}$ inputs) thanks to a novel compensation technique, large tuning range of the transconductance (factor 100 ), floating inputs and a resulting good CMRR.

\section{Introduction}

Linear CMOS voltage to current converters can be classified in two groups: The first group has conversion transistors operating in saturation. The linearization is in most cases obtained by exploiting the transistor's square law behavior [1-3]. The second class of $\mathrm{VI}$ converters has conversion transistors operating in nonsaturation; the triode region [4-6]. The advantages compared to the square-law converters are a higher linearity and a larger tuning range of the transconductance.

This paper describes a novel versatile triode VI converter with floating inputs, having a good linearity and a large transconductance tuning range.
\end{abstract}

\section{Basic principle}

Using a simple model, the drain current of an MOS transistor in triode region can be written as:

$$
\begin{array}{r}
I_{d}=\beta V_{d s}\left(\left(V_{g s}-V_{t}\right)-\frac{1}{2} V_{d s}\right) \\
\text { with } \beta=\frac{\mu C_{o x W}}{L}
\end{array}
$$

If the drain-source voltage $V_{d s}$ is held constant then the triode transistor has a linear $V_{g s}$ to $l_{d}$ transfer.

The most obvious way to keep $V_{d s}$ constant is to add a (folded) cascode transistor to the triode transistor. A problem is now that any $I_{d}$ variation of the triode transistor results in a $V_{g s}$ variation of the cascode transistor. This in turn results in a $V_{d s}$ variation of the triode transistor since the gate of the cascoding device is normally biased at a constant voltage. The result of this is nonlinearity in the voltage to current conversion.

To keep $V_{d s}$ after all constant, feedback is generally applied [4,5] resulting in a constant $V_{d s}$ at least for low frequencies. In reference [6] no special circuitry is added to reduce the variation in $V_{d s}$ but the cascode transistor has a very large aspect ratio.

This paper makes use of an alternative way to keep $V_{d s}$ constant: compensation.

The basic principle is given in figures $1 \mathrm{a}$ and $\mathrm{b}$. In figure $1^{a}$ the triode transistor $M 1$ is cascoded with a transistor M2 operating in saturation. The gate voltage of $\mathrm{M} 2$ is not connected to a static voltage source but to a oynamic bias voltage: a static bias plus a copy of the gate-source voltage of M2; $\mathrm{V}_{\text {bias }}+$ $V_{g s 2}$. The result is that if $V_{g s 2}$ is modulated, $V_{d s 1}$ will remain constant and equal to $V_{\text {bias. }}$.

The question is how to generate this dynamic bias voltage. The solution is shown in figure $1^{b}$. The drain current of $M 1$ is split in two equal parts by $M 2$ and M3. The output current flowing through $M 2$ is therefore duplicated in $\mathrm{M} 3$ and mirrored into the 
a)

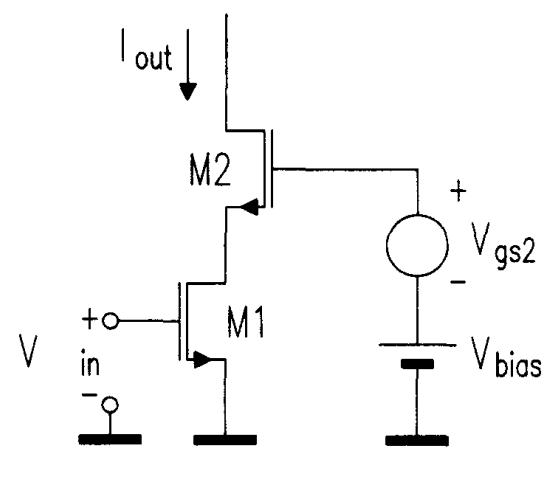

b)

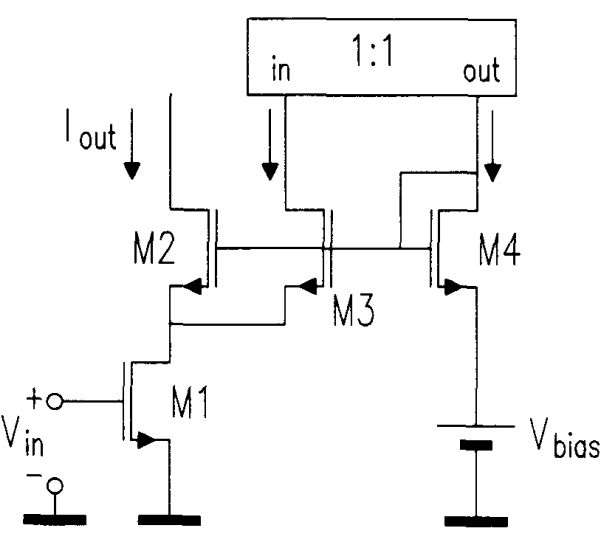

figure 1 a) basic principle of $v_{\mathrm{gs} 2}$ compensation.

b) realization of the dynamic bias voltage

diode connected transistor $M 4$. If $M 3=M 4$ then $V_{g s 3}=$ $V_{g s 4}$, resulting in proper biasing of the gate of $M 2$ as proposed in figure $1^{a}$.

The circuit of figure $1^{b}$ has still several disadvantages: the input is not (truly) floating nor differential and an $A C$ current flows through the bias voltage source. This is overcome in the circuit of figure 2.

\section{The complete circuit}

Differential circuits have better linearity thanks to the cancellation of even order distortion terms. The circuit of figure $2^{a}$ is the differential version of figure $1^{b}$. The triode transistors performing the linear voltage to current conversion are M1A and M1B.
The current mirrors have been replaced by a cross coupled structure with current sources $21_{s}$. The voltage source $V_{\text {bias }}$ of figure $1^{b}$ is replaced by $a$ transistor in triode region (M1C). Thanks to the differential structure the current through $M 1 C$ is constant (see below) and therefore the drain-source voltage of M1C $\left(\mathrm{V}_{\mathrm{ds} 1 \mathrm{C}}\right)$ will also be constant. The drain source voltages of $\mathrm{M} 1 \mathrm{~A}$ and $\mathrm{M} 1 \mathrm{~B}$ are equal to $V_{\text {ds1c }}$ due to the circuit configuration similar to fig. $1^{b} . V_{d s 1 c}$ is set up with the voltage source $V_{b}$ and the current $21_{s}$. Note that when no differential input voltage is present it follows that $V_{g s 1 A}=V_{g s 1 B}=V_{b}$ since $M 1 A=M 1 B=M 1 C$. The transistors $M 1 A, M 1 B$ and $M 1 C$ now all have drain currents equal to $2 \mathrm{I}_{\mathrm{s}}$ and the transistors M2-M4 all have quiescent currents equal to $I_{s}$. The voltage source $V_{b}$ is implemented by means of a diode connected transistor with constant drain current as shown in figure $2^{\mathrm{b}}$. If a positive input voltage is applied to the circuit, the currents in the branches flow as illustrated with arrows. It can be easily argued that the current through $\mathrm{M} 1 \mathrm{C}$ is constant indeed. Note that the input voltage is not restricted to a certain common-mode voltage level; the inputs are really floating resulting in a good CMRR (determined by the output impedances of the current sources).

Keeping $V_{b}$ constant, $V_{d s 1 c}$ can be varied with $I_{s}$. Using equation (1) $V_{d s 1 c}$ can be solved:

$V_{d s 1 c}=\left(V_{b}-V_{t}\right)-\sqrt{\left(V_{b}-V_{t}\right)^{2}-\frac{4 I s}{\beta 1}}$

$V_{d s 1 C}$ equals $V_{d s 1 A}$ and $V_{d s 1 B}$ and thus the transconductance of the $\mathrm{V}-\mathrm{I}$ converter can be written as:

$$
\begin{aligned}
g m= & \frac{I_{\text {out }+}-I_{\text {out }-}}{V_{\text {int }}-V_{\text {in }}}=\frac{1}{2} \cdot \beta_{1} \cdot V_{d s i c}= \\
& \frac{1}{2} \beta_{1}\left(\left(V_{b}-V_{t}\right)-\sqrt{\left(V_{b}-V_{t}\right)^{2}-\frac{4 I_{s}}{\beta 1}}\right.
\end{aligned}
$$

The transconductance can be tuned with the tail current $I_{s}$. If the transistors M1A, M1B and M1C operate in deep triode i.e. $2 l_{s}<\frac{\beta}{2}\left(V_{b}-V_{t}\right)^{2}$ then 


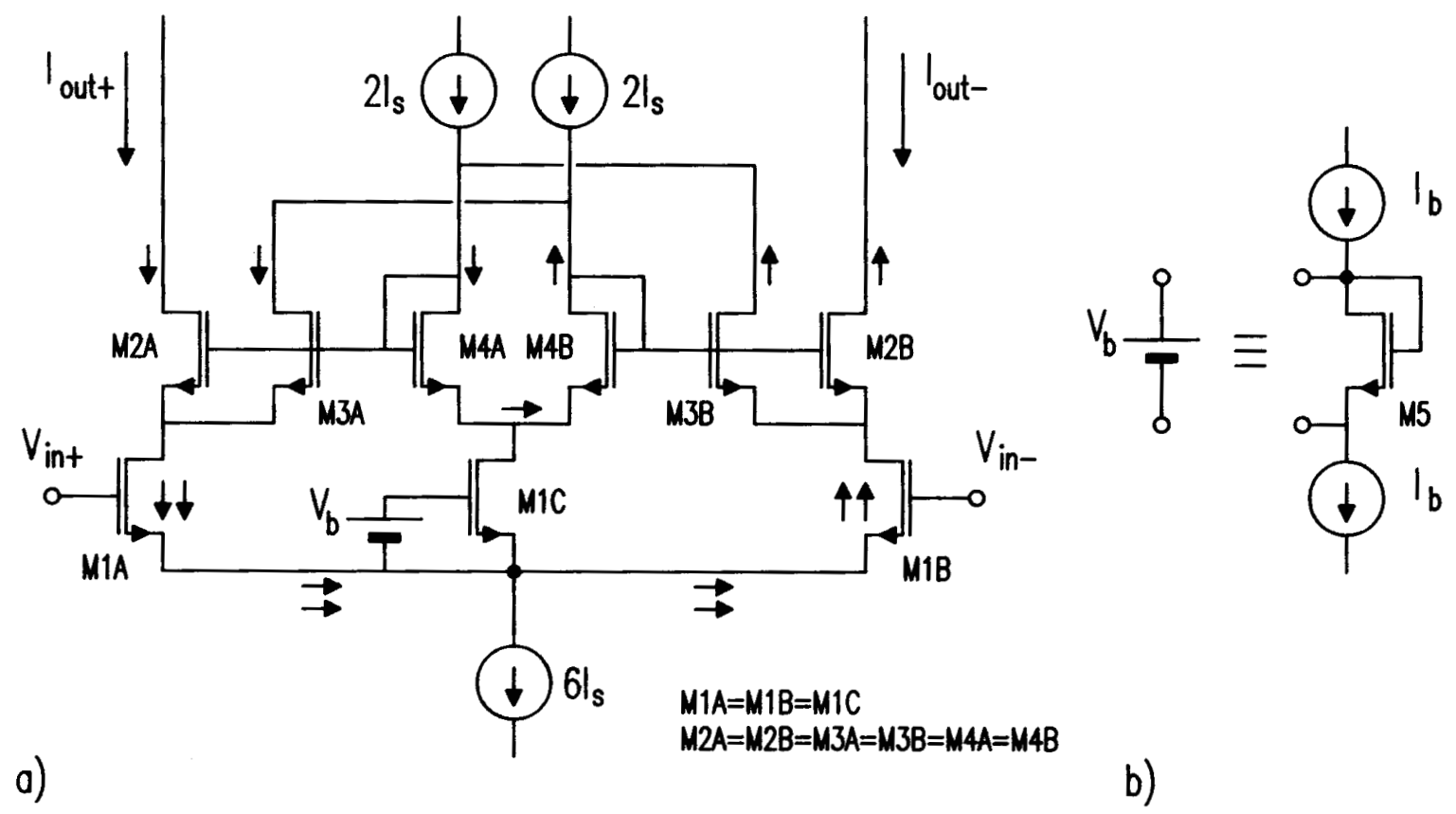

figure 2 a) The complete transconductor circuit.

b) realization of the voltage source $V_{b}$.

expression (3) can be simplified into:

$g m \approx \frac{I s}{\left(V_{b}-V_{t}\right)}$

\section{Distortion}

Although the transconductor is linear according the simple MOS model of equation (1) there will be some small nonlinearities if a more sophisticated model is used. If there is no mismatch in the circuit the main source of distortion will be mobility reduction. ( commonly modeled with $\theta$, typically between 0.05 and $0.5 V^{1}$ ) The distortion is then only odd order distortion since the even harmonics are canceled in a perfect differential structure. It can be shown that the total harmonic distortion is now dominated by the third order term:

$H D 3 \approx \frac{1}{16}\left[\frac{\theta}{\left(1+\theta\left(V_{b}-v_{t}\right)\right.}\right]^{2} \cdot\left|v_{i n}\right|^{2}$

The ripple in $\mathrm{V}_{\mathrm{ds}}$ of $\mathrm{M} 1 \mathrm{~A}$ and $\mathrm{M} 1 \mathrm{~B}$ depends on matching. If the matching of all transistors and current sources is ideal, $V_{d s}$ is perfectly constant and eq.
(5) is valid. A mismatch in $\beta$ and $V_{t}$ will result in a ripple in $V_{d s}$. For very low values of $V_{d s}$ this ripple will cause additional second and third order distortion and therefore a lower limit of the $V_{d s 1}$ and transconductance is imposed. Monte Carlo simulations of the complete circuit (including current sources) show that for a typical $\beta$ mismatch of $1 \%$ and $V_{t}$ mismatch of $1 \mathrm{mV}$ the variation in $V_{d s}$ is several millivolts depending on $I_{s}$. For larger $V_{d s}$ levels $\left(V_{d s}=200 \mathrm{mV}\right)$ the distortion is still mainly third order distortion ( $\mathrm{HD} 3 \approx 0.5 \%$ for $6 \mathrm{~V}_{\mathrm{pp}}$ inputs ). For lower $V_{d s}$ levels $\left(V_{d s}=10 \mathrm{mV}\right)$ the distortion consists of second and third order distortion ( $\mathrm{HD} 2 \approx 0.3 \%$ and $\mathrm{HD} \approx \approx 0.2 \%$ for $6 \mathrm{~V}_{\mathrm{pp}}$ inputs )

\section{Bandwioth}

If, like in equation (4), the transistors $M 1$ are assumed to operate in deep triode, the estimated bandwidth of the circuit becomes:

$\omega_{-3 d B} \approx \frac{2 \cdot r_{d s 1} \cdot g m_{2}^{2}}{3 \cdot C_{g s 2}}$ 
Where $r_{d s 1}$ is the small-signal drain-source resistance of the transistors $M 1, \mathrm{gm}_{2}$ is the transconductance and $\mathrm{C}_{\mathrm{gs} 2}$ is the gate-source capacitance of the transistors M2-M4. Simulations using typical values for a $3 \mu \mathrm{m}$ CMOS process result in $1-70 \mathrm{MHz}$ bandwidth depending on $\mathrm{I}_{\mathrm{s}}$.

\section{Experimental results}

At this moment a test chip is being processed. The experimental results now available were obtained from a breadboard realization built with commercially available CA3600 CMOS-transistor arrays. The transconductance was measured for different tail currents $I_{s}(19 \mu \mathrm{A} .600 \mu \mathrm{A})$ and corresponding different values of $V_{d s}(21 \mathrm{mV} . .672 \mathrm{mV})$. The voltage $V_{b}$ was chosen equal to $V_{t}+2 V$. The results are given in figure 3. The nonlinearities are mainly due to mobility reduction and mismatch. As expected the effects of mismatch dominate at small $V_{d s}$ values. The transconductance was tuned from $10 \mu \mathrm{A} / \mathrm{V}$ to $250 \mu \mathrm{A} / \mathrm{V}$, which is a factor 25 in transconductance. An on-chip realization will have a larger tuning range owing to the better matching on chip. Simulations, taking account of mismatch, predict a factor 100 transconductance tuning range. The measured THD was less than $0.4 \%$ for $6 V_{p p}$ input voltages $\left(V_{d s}=336 \mathrm{mV}\right)$. This THD consists of mainly second harmonics caused by mismatch.

gm [UAN]

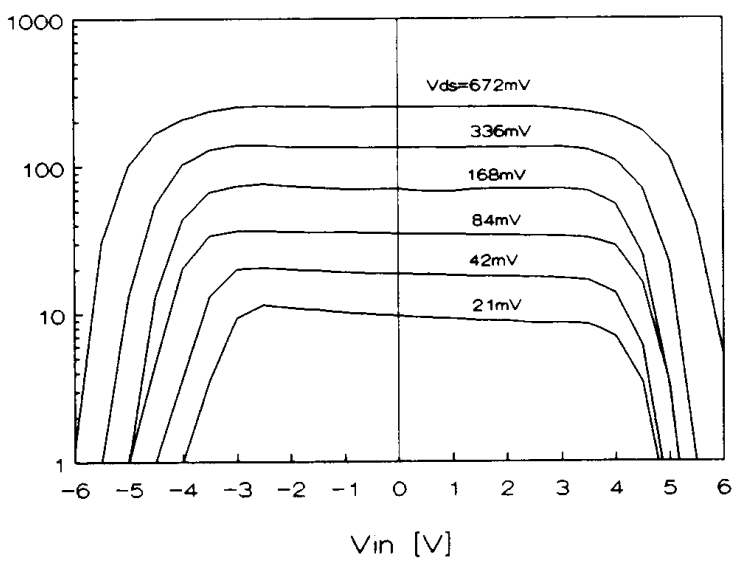

\section{Conclusions}

A Novel transconductor is presented. The linearity is high (THD $<0.4 \%$ for $6 \mathrm{~V}_{\mathrm{pp}}$ input signals on a breadboard) The transconductance can be tuned over a wide range (factor 25 on breadboard, factor 100 expected on chip). The input voltages are not restricted to a common mode level; the transconductor has floating inputs resulting in a good CMRR.

Applications can be found in the field of OTAs, (programmable) filters, (tunable) amplifiers, automatic gain control etc.

\section{acknowledgment}

The authors wish to thank R.F. Wassenaar and H. Wallinga for fruitful discussions. This work was sponsored by the Dutch IOP (innovative research projects program) program.

\section{References}

[1] Nedungadi, A. P., and Viswanathan, T. R.: "Design of linear transconductance elements", IEEE Trans. Circuits Syst., 1984, CAS-31, pp 891-894.

[2] K. Bult and $H$. Wallinga, "A Class of analog CMOS circuits based on the square law characteristic of a MOST in saturation" IEEE J. Solid State Circ., SC-22, pp 357-365.

[3] E. Seevinck and R.F. Wassenaar, "A versatile CMOS linear transconductor/ square law function circuit", IEEE J. Solid State Circ. SC-22, pp 366-377.

[4] Pennock, J.L. "CMOS triode transconductor for continuous-time active integrated filters" Electr. Lett. Vol 21, 1985 pp 817-818

[5] Gatti, U., Maloberti, F., Torelli, G., "A novel CMOS linear transconductance cell for continuous-time filters", ISCAS'90 pp 1173-1176.

[6] Stefanelli, B, and Kaiser, A, "CMOS Triode transconductor with high dynamic range" Electr. Lett. 21 june 1990, Vol.26 No.13, pp 880-881.

Figure 3 Measured transconductance versus $\longleftarrow$ differential input voltage $\left(V_{\text {in }}=V_{\text {in }+}-V_{\text {in- }}\right)$ for several values of $V_{\mathrm{ds}}(i s)$. (breadboard) 